\title{
TUMORES VESICALES INUSUALES: CARCINOMA EPIDERMOIDE, ADENOCARCINOMA Y SARCOMA PRIMARIOS. COMPORTAMIENTO CLÍNICO. NUESTRA EXPERIENCIA
}

\author{
J.A. QUEIPO ZARAGOZÁ, F. CHICOTE PÉREZ, A. BORRELL PALANCA, \\ J.F. BELTRÁN MESEGUER, C. ALCALÁ-SANTAELLA CASANOVA, \\ B. MARTÍNEZ GARCÍA, F. PASTOR SEMPERE
}

Servicio de Urología. Hospital de Sagunto. Valencia.

Actas Urol Esp. 27 (2): 123-131, 2003

\section{RESUMEN}

“TUMORES VESICALES INUSUALES: CARCINOMA EPIDERMOIDE, ADENOCARCINOMA Y SARCOMA PRIMARIOS. COMPORTAMIENTO CLÍNICO. NUESTRA EXPERIENCIA"

INTRODUCCIÓN Y OBJETIVOS: Los tumores vesicales con un patrón morfológico no transicional, constituyen un reto diagnóstico al patólogo y terapéutico al urólogo, pues aunque son poco frecuentes (menos del 5\% de los tumores malignos de vejiga), presentan un comportamiento biológico diferente que obliga a un abordaje terapéutico particular. Las variedades anatomopatológicas más importantes son: el carcinoma epidermoide, el adenocarcinoma y el sarcoma primarios. Analizamos nuestra experiencia en este tipo de tumores.

MATERIAL Y MÉTODOS: Estudio retrospectivo de los tumores vesicales inusuales tratados en nuestro Hospital entre 19882001. Analizamos su comportamiento biológico y tratamiento aplicado. En todos ellos se descartó el origen extravesical de la tumoración. RESULTADOS: Encontramos 21 casos, cuyas variedades anatomopatológicas fueron: 13 Carcinomas Epidermoides, 7 Adenocarcinomas (3 de Uraco) y un Sarcoma. La edad media fue muy diferente entre los carcinomas epidermoides $(69,2 \pm 9,1$ años) y los adenocarcinomas $(50,3 \pm 8,1)$. En las tres variedades histológicas el comportamiento fue muy agresivo. En el momento del diagnóstico 19 pacientes presentaban estadios locales avanzados ( $\geq \mathrm{T} 2)$. Aunque el tratamiento electivo fue la cistectomía, sólo se pudo realizar en 10 casos. Quimioterapia sistémica (la más frecuente M-VAC) y/o RT local se aplicó en 7 casos. 14 pacientes han fallecido, con una supervivencia media de 15,7 $\pm 11,6$ meses.

CONCLUSIONES: El diagnóstico tardío de estos tumores y su agresivo comportamiento biológico comportan un pronóstico sombrío. Sólo un diagnóstico precoz y un tratamiento radical podrían mejorar su pronóstico.

PALABRAS CLAVE: Tumor vesical. Carcinoma epidermoide. Adenocarcinoma. Sarcoma.

\section{ABSTRACT \\ "UNUSUAL TUMOURS OF THE BLADDER: PRIMARY EPIDERMOID CARCINOMA, ADENOCARCINOMA AND SARCOMA. CLINICAL BEHAVIOUR. OUR EXPERIENCE"}

INTRODUCTION AND OBJECTIVES: Non-transitional cell tumours of the bladder are both a diagnostic challenge for the pathologist and a therapeutic challenge for the urologist, because although uncommon (less than $5 \%$ of all malignancies of the bladder) they show different biological behaviours each requiring a unique approach. The most significant pathoanatomical types are: primary epidermoid carcinoma, primary adenocarcinoma and primary sarcoma. This paper presents an analysis of our experience in these types of tumours.

MATERIAL AND METHODS: A retrospective study of unusual cases of cancer of the bladder seen in our hospital between 1988-2001. Their biological behaviour and the therapies applied are analysed. The extravesical origin of the tumour was ruled out in all cases.

RESULTS: We found 21 cases of the following pathoanatomical varieties: 13 epidermoid carcinomas, 7 adenocarcinomas (3 urachal) and 1 sarcoma. Mean age was very different between epidermoid carcinomas (69.2 \pm 9.1 years) and adenocarcinomas $(50.3 \pm 8.1)$. Tumour behaviour was very aggressive in all three histological varieties. At the time of diagnosis 19 patients had locally advanced stages ( $\geq \mathrm{T} 2)$. Although elective therapy was cystectomy, this was only feasible in 10 cases. Systemic chemotherapy (most frequently M-VAC) and/or local radiotherapy was used in 7 cases. 14 patients have died after a mean survival of $15.7 \pm 11.6$ months.

CONCLUSIONS: Late diagnosis of these tumours and their aggressive biological behaviour involve a gloomy prognosis. Only early diagnosis and radical therapy could improve the prognosis.

KEY WORDS: Tumour of the bladder. Epidermoid carcinoma. Adenocarcinoma. Sarcoma. 


\section{INTRODUCCIÓN Y OBJ ETIVOS}

Los tumores vesicales inusuales podemos definirlos como aquellos tumores que presentan un patrón morfológico diferente al transicional, aunque en algunos casos aparezcan asociadas áreas tipicas de carcinoma transicional (formas mixtas). Estos tumores suponen un reto tanto diagnóstico para el patólogo como terapéutico para el urólogo, pues aunque son poco frecuentes (constituyen menos del 5\% de los tumores malignos de vejiga), presentan dificultades de clasificación anatomopatológica y un comportamiento clínico diferente, que determina una particular actitud terapéutica. Las variedades anatomopatológicas más frecuentes son: el carcinoma epidermoide, el adenocarcinoma y el sarcoma primarios. Su histogénesis es motivo de discusión, atribuyéndose a células de extirpe transicional o no; en cualquier caso, bien a partir de la metaplasia de células transicionales o bien a partir de células pluripotenciales presentes en la mucosa vesical, se produce la diferenciación a células escamosas a células cúbicas de naturaleza glandular, dando lugar al carcinoma epidermoide o al adenocarcinoma respectivamente. En el caso del sarcoma su origen es el tejido conjuntivo-mesenquimal. El objetivo de este trabajo es analizar nuestra experiencia en este tipo de tumores y una revisión de la literatura.

\section{MATERIAL Y MÉTODOS}

Hemos realizado un estudio retrospectivo de los tumores vesicales diagnosticados y tratados en el Servicio de Urología de nuestro hospital, desde 1988 hasta la actualidad. El total de tumores vesicales ha sido de 429. Para la selección de los casos motivo de estudio, se eligieron aquellos que presentaban un patrón anatomopatológico de predominio no transicional, descartando además el origen extrínseco de la tumoración (donde predominó el origen intestinal, ovárico y prostático). Tras la revisión por el patólogo, el total de casos seleccionados fue de 21 , que correspondieron a 13 carcinomas epidermoides, 7 adenocarcinomas y 1 sarcoma. Del total de carcinomas epidermoides, 5 fueron formas puras y 8 tumores mixtos de predominio epidermoide (con áreas aisladas de carcinoma transicional). De los 7 adenocarcinomas, 3 fueron de localización uracal. Sobre esta serie analizamos parámetros epidemiológicos, compor- tamiento clínico, tratamiento aplicado y evolución. La supervivencia se analizó mediante curvas de Kaplan-Meier (Log-rank).

\section{RESULTADOS}

\section{Datos epidemiológicos}

De los 21 casos, sólo 3 correspondieron a mujeres ( 2 carcinomas epidermoides y un adenocarcinoma). Destacó la diferencia en la edad media de los adenocarcinomas (50,3 \pm 8, 1 años; Rango: 4457 , muy por debajo de la de los carcinomas epidermoides (69,2 \pm 9, 1; Rango: 50-82). El paciente con sarcoma vesical tenía 82 años.

\section{Factores etiológicos o de riesgo}

Destacó el tabaco. De los 13 pacientes con carcinoma epidermoide, 10 eran fumadores (2 de ellos presentaban además sondaje vesical de larga evolución e infecciones urinarias de repetición), uno presentaba irradiación pélvica previa por un linfoma no Hodking (en remisión completa) y los 2 restantes no presentaron factores de riesgo. En el grupo de adenocarcinomas sólo el tabaco aparecía como factor de riesgo, en 4 casos. El paciente afecto de sarcoma también era fumador.

\section{Sintomatología clínica}

La hematuria fue el motivo de consulta predominante, siendo también frecuente la sintomatología irritativa-miccional. Los pacientes con carcinoma epidermoide consultaron en 6 casos por hematuria macroscópica, en 5 por sintomatología miccional y en 2 casos el hallazgo fue casual. En el grupo de adenocarcinomas, la hematuria dirigió el diagnóstico en 4 pacientes y el sindrome miccional en 2. El paciente afecto del sarcoma consultó por molestias miccionales de predominio irritativo.

\section{Localización, Tamaño y Estadiaje}

La localización predominante en los carcinomas epidermoides fue la base vesical (trígono y retrotrígono), presentando esta localización el 85\% de casos; ello determinó que el $62 \%$ de pacientes presentaran ureterohidronefrosis uni o bilateral en distintos grados. 3 adenocarcinomas se localizaron en Uraco, y los 4 restantes en pared lateral derecha, pared lateral izquierda, trígono y retrotrígono respectivamente. El sarcoma se localizó en la cúpula vesical. 
Respecto al tamaño tumoral en el momento del diagnóstico predominaron los tumores de gran tamaño; sólo 2 pacientes presentaban tumores $<3$ $\mathrm{cm}$ ( 2 tumores epidermoides) y en 11 casos el tumor fue $>5 \mathrm{~cm}$ (6 carcinomas epidermoides, 4 adenocarcinomas y el sarcoma).

19 pacientes se presentaron en estadios clínicos avanzados ( $\geq \mathrm{T} 2)$. En el grupo de carcinomas epidermoides predominó el estadio T4, presentando 3 pacientes adenopatías en el momento del diagnóstico y uno metástasis; en la serie de adenocarcinomas predominó el T3, con adenopatías en un caso y metástasis en 2 . El sarcoma presentó un estadio T3bNoMo (Gráfico 1).

\section{Tratamiento}

El tratamiento electivo fue la cistectomía, dado el estadiaje y agresividad de estos tumores; pero sólo se pudo realizar en 10 pacientes, por la presencia de factores que la contraindicaban: edad avanzada, irresecabilidad del tumor, presencia de metástasis, etc. El tratamiento realizado en cada tipo tumoral se representa en la Tabla I.

En el grupo de carcinomas epidermoides sólo se realizó cistectomía en 6 pacientes (46\%); en 2 casos se realizó Quimioterapia sistémica (Esquema M-VAC) y en 5 pacientes se hizo únicamente RTUs paliativas ( 3 por su edad avanzada, uno por la irresecabilidad del tumor y otro por la presencia de metástasis).

En el grupo de adenocarcinomas, no se pudo realizar cistectomía en 2 pacientes (por la presencia de metástasis), sometiendo uno de ellos a quimioterapia con el Esquema M-VAC y el otro con Esquema FAC. Un paciente se sometió únicamente a RTU por la irresecabilidad del tumor y en los 4 restantes se realizó cistectomía (una de ellas parcial).

Destacó que de los 10 pacientes sometidos a cistectomía, en 4 casos los bordes quirúrgicos pre-

\section{EPIDERMOIDE}

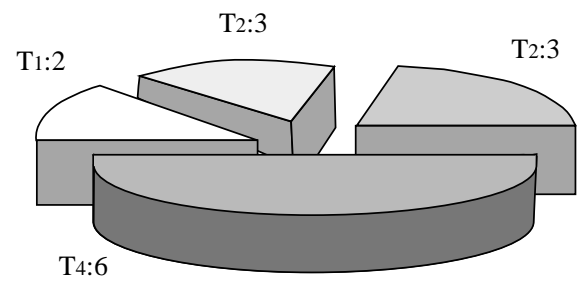

\begin{tabular}{|lll|}
\hline No:9 & N1:3 & N2:1 \\
\hline M0:12 & M1:1 & \\
\hline
\end{tabular}

ADENOCARCINOMA

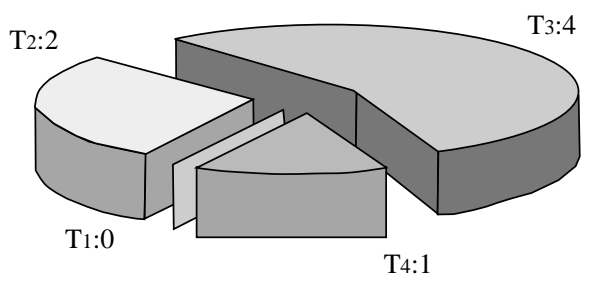

\begin{tabular}{|lll|}
\hline No:6 & N1:0 & N2:1 \\
\hline M0:5 & M1:2 & \\
\hline
\end{tabular}

GRÁFICO 1. Distribución de casos por TNM.

TABLA I

TRATAMIENTO

\begin{tabular}{|l|c|c|c|}
\hline $\begin{array}{l}\text { Tratamiento inicial/ } \\
\text { Tipo tumoral }\end{array}$ & $\begin{array}{c}\text { Carcinoma } \\
\text { epidermoide }\end{array}$ & Adenocarcinoma & Sarcoma \\
\hline RTU radical & 5 & 1 & 1 \\
\hline Cistectomia & 6 & $4 *$ & - \\
\hline RTU + QT & 2 & 2 & - \\
\hline
\end{tabular}

*Una de ellas fue cistectomía parcial.

**En 4 pacientes los bordes quirúrgicos presentaban tumor. 
sentaban tumor, aplicando en 3 de ellos ( 2 adenocarcinomas y un carcinoma epidermoide) quimioterapia adyuvante. Radioterapia con intención hemostática y antiálgica se realizó en 4 pacientes.

\section{Evolución y Supervivencia}

El tiempo de recidiva local para el carcinoma epidermoide fue de 4,3 $\pm 3,1$ meses (Rango: 0-9) y de 7,0 $\pm 10,3$ meses (Rango: 0-27) para el adenocarcinoma. El tiempo medio de aparición de metástasis fue de 11,4 \pm 6,6 meses (Rango: 0-19) para el carcinoma epidermoide y de 13,0 $\pm 15,7$ meses (Rango: 0-43) para el adenocarcinoma. En el caso del sarcoma las metástasis aparecieron a los 5 meses del diagnóstico.

La supervivencia actuarial global es de $24,4 \pm$ 25,1 meses (rango: 2-108) (Gráficos 2 y 3). Han

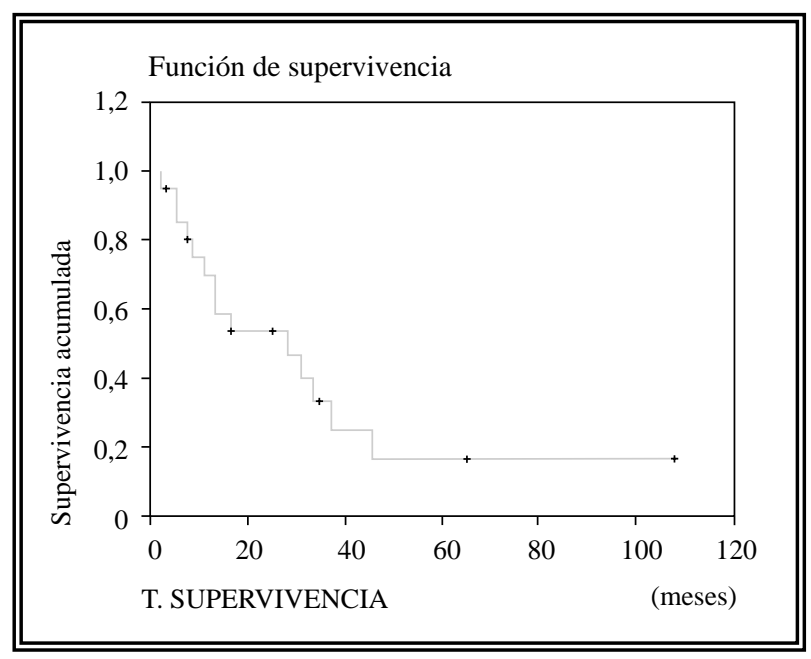

GRÁFICO 2. Curva de Kaplan-Meier del conjunto de tumores.

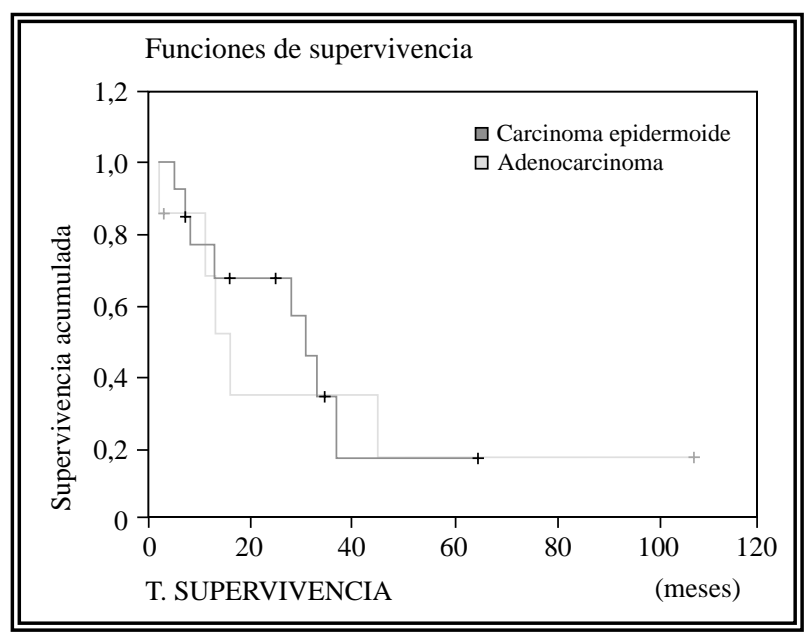

GRÁFICO 3. Curvas de Kaplan-Meier: carcinoma epidermoide vs adenocarcinoma. fallecido 14 pacientes, todos ellos inicialmente infiltrantes, con una supervivencia media de 15,7 \pm 11,6 meses. El paciente afecto de sarcoma falleció a los 6 meses del diagnóstico. De los 13 éxitus restantes, 8 fueron carcinomas epidermoides y 5 adenocarcinomas. La supervivencia actuarial para cada grupo anatomopatológico fue: carcinomas epidermoides 23,9 $\pm 17,1$ meses (Rango: 5-65) $\sin$ diferencias significativas entre los tumores puros y aquellos con áreas de carcinoma transicional. En los adenocarcinomas la supervivencia actuarial fue de 28,3 \pm 38 ,0 meses (Rango: 2-108) sin encontrar tampoco diferencias estadísticamente significativas entre los carcinomas de uraco y el resto (Gráficos 4 y 5).

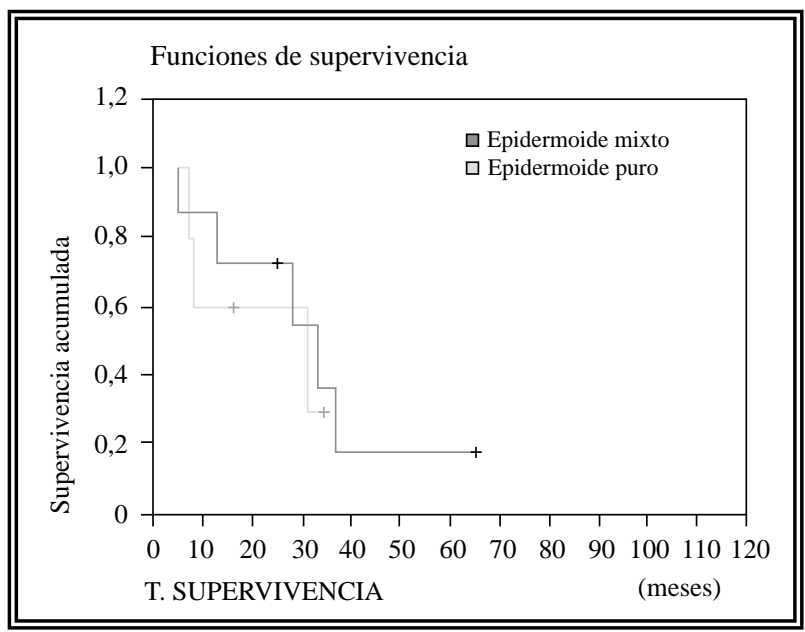

GRÁFICO 4. Curvas de Kaplan-Meier: carcinoma epidermoide puro vs mixto.

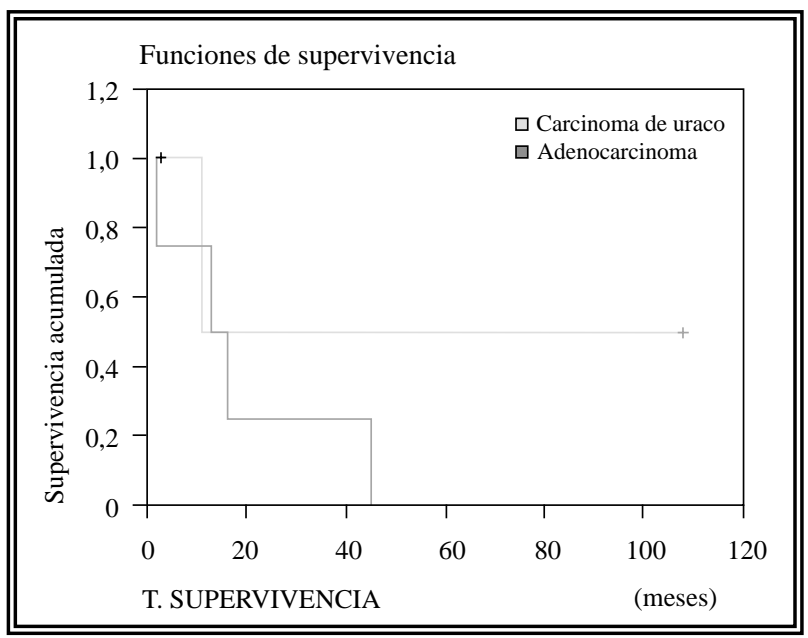

GRÁFICO 5. Curvas de Kaplan-Meier: carcinoma de uraco us resto de adenocarcinomas 


\section{DISCUSIÓN}

\section{Carcinoma epidermoide}

Los carcinomas epidermoides de vejiga son tumores poco frecuentes en nuestro medio, pues suponen únicamente entre el $3-6 \%$ de los tumores vesicales ${ }^{1-3}$; sin embargo su frecuencia si es alta en otras zonas donde la infección por Schistosoma haematobium (directamente relacionado con su génesis) es endémica ${ }^{4}$. Lo que sí es frecuente en nuestro medio es la presencia de áreas de diferenciación escamosa en el seno de un carcinoma transicional. De todas formas, es el tumor con patrón anatomopatológico no transicional más frecuente. En nuestra serie ha representado el 3\% de todos los tumores vesicales y el $62 \%$ de los tumores con patrón histológico inusual.

En cuanto a su etiología, además de la relación con la esquistosomiasis, aparecen otros factores implicados, generalmente agentes que provocan irritación vesical crónica (infecciones, sonda vesical permanente, litiasis, retención crónica de orina, etc.), sin olvidar el tabaco ${ }^{1,5-7}$. En Egipto el $75-84 \%$ de casos están en relación con la infección por Schistosoma haematobium y en África el carcinoma epidermoide representa el 53\% de todos los tumores vesicales, frente a sólo el $2 \%$ en las áreas de raza caucasiana ${ }^{1}$.

Su histogénesis está todavía en discusión ${ }^{8}$; la teoría mas aceptada es la formación a partir de tumores uroteliales metaplásicos de larga evolución ${ }^{3}$. Navon JD y col. ${ }^{9}$ describe las mismas aberraciones cromosómicas que las presentes en los carcinomas transicionales. Los que ponen en entredicho esta teoría alegan no apreciar en el tumor focos de epitelio transicional neoplásico.

Son más frecuentes en varones respecto de mujeres, pero en menor proporción que los carcinomas transicionales y se presentan entre la sexta y séptima décadas de la vida, con una edad media de 67 años. La hematuria y disuria aparecen como los principales síntomas de inicio y la localización trigonal la más frecuente, presentándose de gran tamaño en el momento del diagnóstico (tamaño medio de $5 \mathrm{~cm})^{1}$. Por ello es característica la enfermedad local, con extensión perivesical y estadios avanzados. En la serie de Debbagh A y col. ${ }^{10}, 11$ de 14 pacientes presentaban obstrucción del tracto urinario superior y en 10 casos el tumor era pal- pable por tacto rectal. Tanto la afectación linfática como las metástasis a distancia son poco frecuentes y de aparición tardía ${ }^{3}$.

Histológicamente está formado por nidos de células malignas con grados variables de queratinización (Figs. 1 y 2). La característica inmunohistoquímica que lo define es el patrón de citoqueratinas. En los tumores epidermoides predominan las citoqueratinas de alto peso molecular frente a las de bajo peso molecular en los carcinomas transicionales ${ }^{11}$. El CEA es negativo en los carcinomas epidermoides y en los uroteliales presenta una tinción muy débil ${ }^{12}$. Por tanto podemos diferenciar dos grupos, los tumores epidermoides puros y los tumores mixtos, presentando peor comportamiento clínico las formas puras respecto de los carcinomas transicionales con focos de diferenciación escamosa (supervivencia media de 12,5 meses en el carcinoma puro frente a 26,55 en el mixto) ${ }^{13}$.

El tratamiento de elección es la cistectomía radical, no contraindicándola la existencia de adenopatias ${ }^{14}$. La eficacia de la Guimioterapia (QT) y Radioterapia (RT) está en discusión. Algunos autores mejoran los resultados administrando QT y RT preoperatoria ${ }^{15-18}$.

La supervivencia llega a ser de sólo el $12,5 \%$ a los 2 años y del $0 \%$ a los 5 años $^{2,3}$, si bien otros autores como Ghoneim y col. ${ }^{14}$ sobre una serie amplia de 1.026 cistectomías (59\% de casos por carcinoma escamoso) en una zona endémica, alcanza una supervivencia a los 5 años del $46 \%$ de pacientes.

\section{Adenocarcinoma}

Los adenocarcinomas vesicales representan entre el 0,5 y $2 \%$ de los tumores malignos de vejiga $^{19}$. Respecto a su origen se ha especulado mucho, ya que el urotelio carece de epitelio glandular, describiéndose diversas teorías; así tendríamos los adenocarcinomas que se originan a partir de restos uracales, los originados a partir de restos mesonéfricos presentes en la base vesical y los originados a partir de células pluripotenciales incluidas en el epitelio transicional; algunos autores consideran también el origen a partir de cambios metaplásicos de las propias células transicionales, de manera que las glándulas de VonBrunn, constituidas por invaginación submucosa 


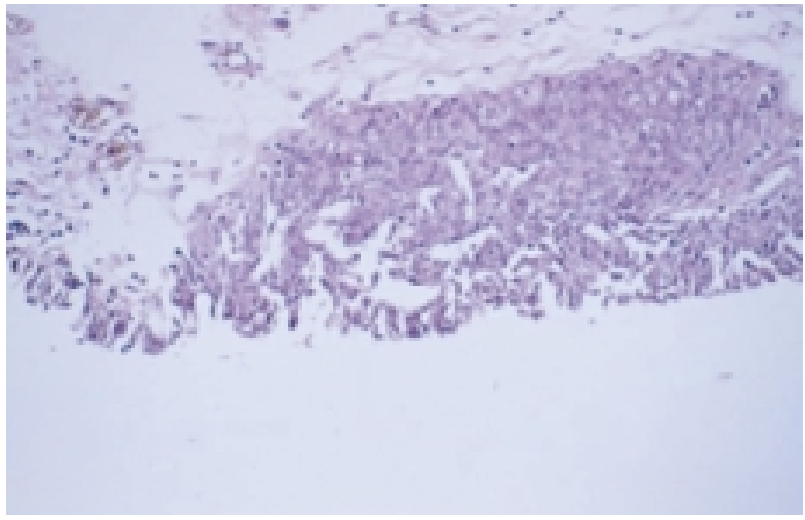

FIGURA 1. Área de metaplasia escamosa. H-E 66x.

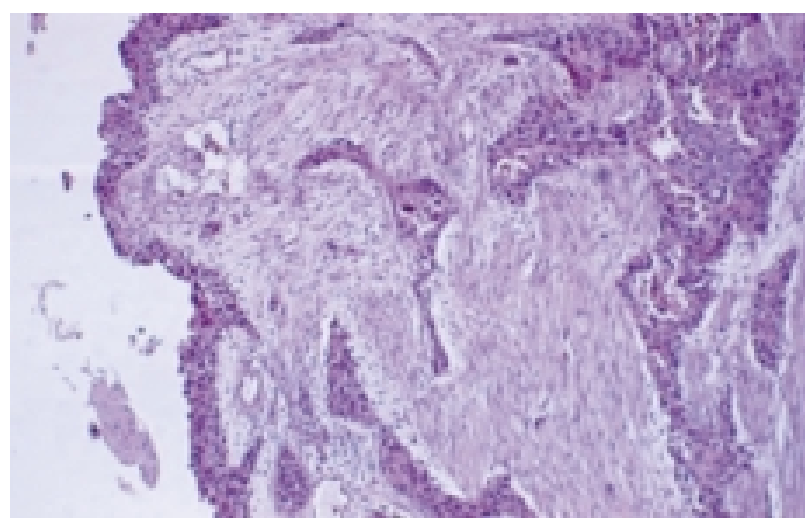

FIGURA 2. Carcinoma epidermoide (estroma queratinizado). H-E 33x.

del propio urotelio, o la cistitis glandular, podrían ser consideradas como lesiones premalignas ${ }^{20}$.

En el planteamiento diagnóstico de estos tumores es necesario el despistaje de aquellos tumores extrínsecos de extirpe glandular que por continuidad o metástasis invaden la vejiga (hecho mucho más frecuente que el origen primario); suelen ser tumores de origen prostático, ginecológico (ovario) o intestinal (colon) ${ }^{21}$. En la serie de Sánchez Salabardo D y col. ${ }^{21}$, de 33 adenocarcinomas vesicales sólo 5 fueron primarios.

Aunque su subclasificación es controvertida, podemos distinguir diversos subtipos: adenocarcinomas de origen uracal, adenocarcinomas mucinosos, adenocarcinomas de células en anillo de sello y tumores mixtos (asociados a áreas de carcinoma transicional).

Los tumores "de origen uracal" se originan a partir de restos del Uraco (resto embrionario fibromuscular en forma de cordón que se extiende desde la cúpula vesical hasta el ombligo, histoló- gicamente formado por tejido fibroconectivo y muscular asociado a epitelio mixto urotelial e intestinal-cloacal). Por ello su localización es la cúpula vesical o pared anterior de la vejiga y se presentan como una masa suprapúbica por debajo del epitelio vesical (Fig. 3). Estos tumores suponen menos del $1 \%$ de los tumores vesicales malignos y entre el $20-39 \%$ de los adenocarcinomas vesicales $^{21,22}$.

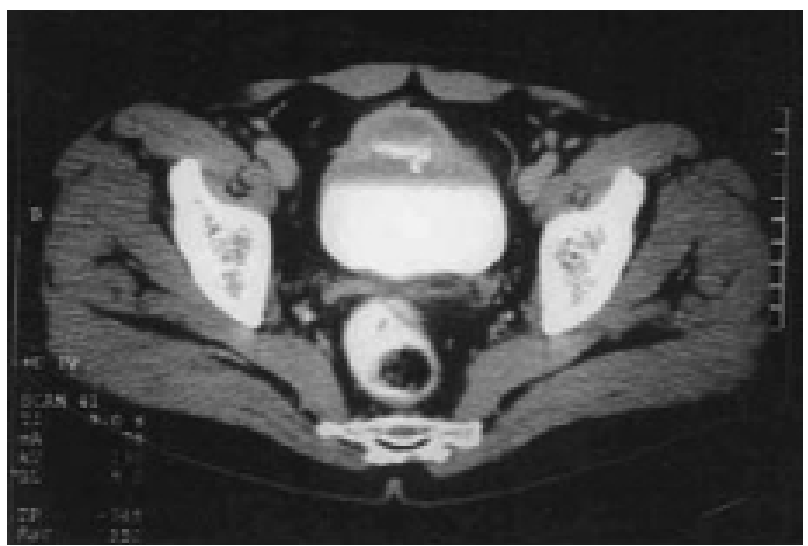

FIGURA 3. TAC (Adenocarcinoma de uraco).

Los tumores "mucinosos" constituye el 0,5-2\% de los tumores vesicales malignos ${ }^{23}$. Se caracterizan por la presencia de las denominadas "células en anillo de sello" o "células claras" (células con un gran citoplasma claro relleno de mucina que desplaza de forma excéntrica un núcleo pleomórfico) y abundante mucina extracelular. En la variedad de "células en anillo de sello", no existe una producción extracelular como ocurre en la modalidad anterior (Figs. 4 y 5).

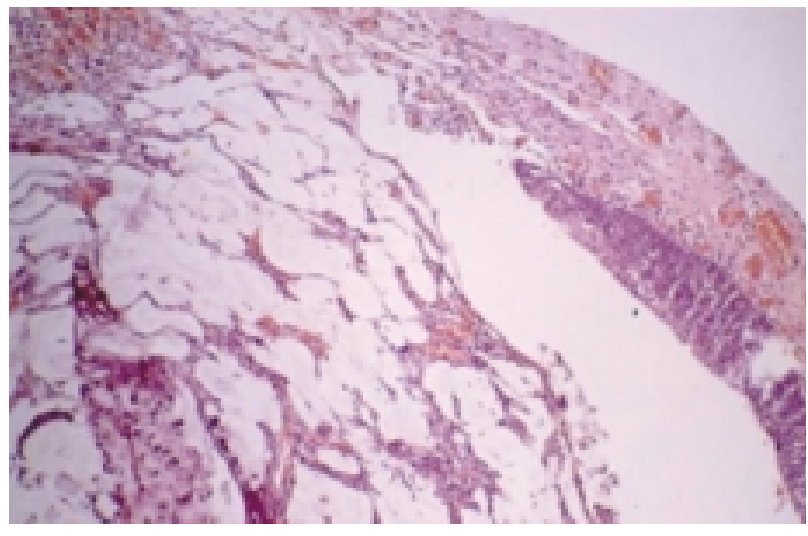

FIGURA 4. Adenocarcinoma mucinoso (Urotelio normal). H-E 33x. 


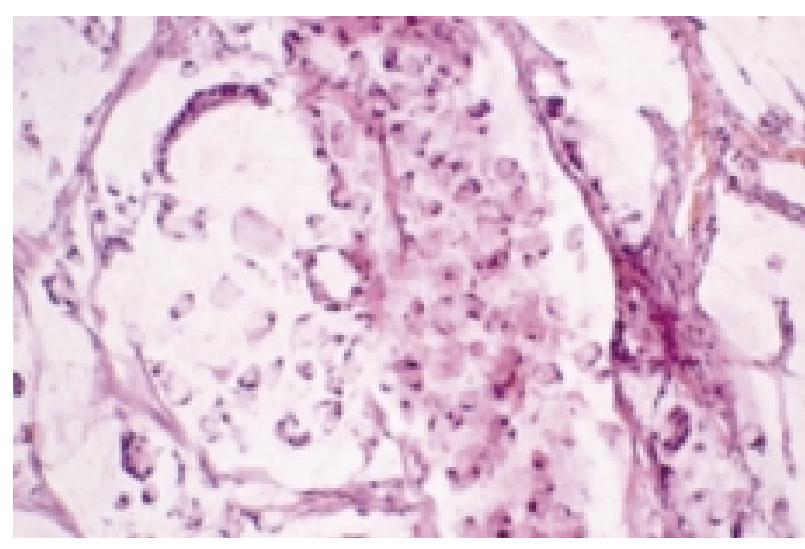

FIGURA 5. Células en anillo de sello y mucina. H-E 120x.

Suelen presentarse con una sintomatología irritativa-miccional florida, si bien la hematuria sigue siendo el sintoma princeps ${ }^{24,25}$. La edad de presentación más frecuente se sitúa entre los 45 y 60 años.

El diagnóstico en estadios avanzados es habitual $^{25}$, lo cual determina su mal pronóstico, sea cual sea su tratamiento. Aproximadamente el 25\% de los pacientes presentan metástasis en el momento del diagnóstico ${ }^{26}$, falleciendo durante el primer año el 60\% de $\operatorname{casos}^{27}$. De las 3 modalidades histológicas la que peor pronóstico presenta es la variedad "en anillo de sello"28,29.

El tratamiento de elección es la cirugía radical (cistectomía) ${ }^{28,29}$, pudiendo en los de origen uracal realizar una cistectomía parcial con amplios márgenes. No hay diferencias de supervivencia en las distintas modalidades de tratamiento (cirugia radical, quimioterapia y radioterapia) cuando el tumor se presenta en estadios avanzados.

Aunque algunos autores no encuentran diferencias de supervivencia respecto de los carcinomas transicionales ${ }^{30}$, en series amplias ${ }^{31}$ las supervivencias alcanzadas llegan a sólo el 11\% a los 5 años.

\section{Sarcoma}

Son escasos los casos publicados, pues suponen menos del $1 \%$ de las neoplasias vesicales ${ }^{32,33}$. $\mathrm{Su}$ origen es mesenquimal, pudiendo un mismo tumor presentar varios patrones histológicos: fibrosarcoma, condrosarcoma, osteosarcoma, leiomiosarcoma etc. ${ }^{34}$ (Figs. 6 y 7).

El diagnóstico en ocasiones no es fácil, siendo necesario distinguirlo del tumor urotelial pseudo-

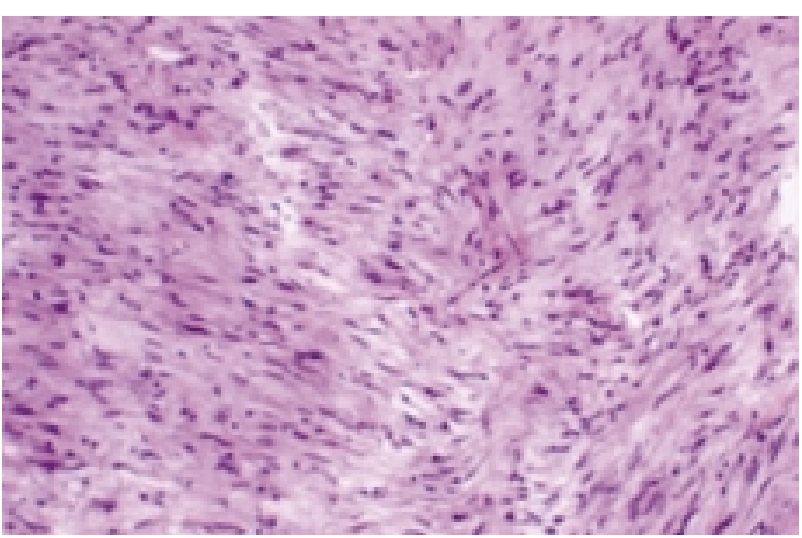

FIGURA 6. Sarcoma vesical; área de fibrosarcoma. H-E 66x.

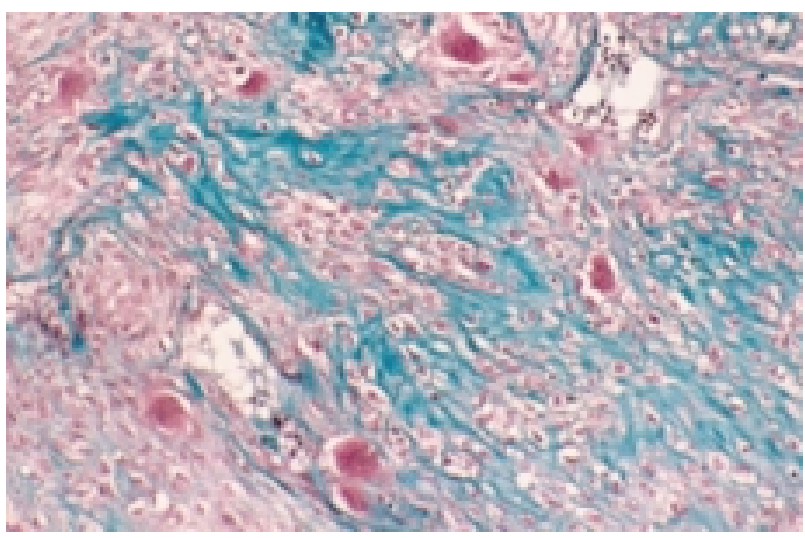

FIGURA 7. Sarcoma vesical (Área de osteosarcoma): Osteoblastos (células pequeñas) Osteoclastos (células grandes). TRICROMICRO DE MASSON 66x.

sarcomatoso, dada la capacidad del urotelio neoplásico de desarrollar cambios metaplásicos condroides u osteoides. El origen mesenquimal de estos tumores se pone de manifiesto con la vimentina (inmunohistoquimia).

Es más frecuente en varones (1.8:1), con una edad media similar a la de los carcinomas vesicales habituales. Los motivos de consulta más frecuentes son la hematuria y la sintomatología miccional. No existe una localización vesical predominante, si bien son frecuentes en la base vesi$\mathrm{cal}^{35}$.

Su pronóstico es infausto, con escasas supervivencias, dado que suelen ser infiltrantes en el momento del diagnóstico y que es frecuente su diseminación local y a distancia vía hematógena. En la serie de Ikegami H y col. ${ }^{35}$, sólo 2 de 14 casos eran superficiales en el momento del diagnóstico. 
El tratamiento de elección sigue siendo la cistectomía radical, pero con pobres resultados ${ }^{32,36}$. Para Oliver C y col. ${ }^{33}$ y Giannopoulos A y col. ${ }^{37}$ la supervivencia se sitúa en torno al $20 \%$ a los 5 años, pero la mayoría fallecen en los primeros 2 años $^{36}$. Ikegami $\mathrm{H}$ y col. ${ }^{35}$ obtienen una supervivencia media de 14 meses y mediana de 7 . No existe consenso respecto de la efectividad de la asociación de quimioterapia o radioterapia.

\section{CONCLUSIONES}

Los tumores vesicales de extirpe metaplásica o no transicional presentan un comportamiento biológico muy agresivo que comporta un pronóstico sombrío. Se presentan en diferentes rangos de edad respecto de los carcinomas transicionales (edades más tardías en los carcinomas epidermoides y más jóvenes en los adenocarcinomas). Aunque la hematuria es la forma más frecuente de presentación, el síndrome miccional también es frecuente. El diagnóstico es tardío, descubriéndose como tumores de gran tamaño, en estadios localmente avanzados, con recidivas y metástasis precoces. El tratamiento de elección es la cistectomía, cuando ésta es factible, con escasa respuesta a la Quimioterapia o Radioterapia. El abordaje mediante RTU queda relegado al diagnóstico histopatológico inicial o a un tratamiento puramente paliativo. Su escasa supervivencia pone de manifiesto la necesidad de un diagnóstico más precoz y un tratamiento agresivo.

\section{REFERENCIAS}

1. SERRETTA V, POMARA G, PIAZZA F GANGE E.: Pure squamous cell carcinoma of the bladder in western countries. Eur Urol 2000; 37: 85-89.

2. SCHRODER MD, WEISS MD, HUGHES MS.: Squamous cell carcinoma of the bladder: an increased incidence in the blacks. Urology 1986; 28: 288291.

3. ZERBIB M, TURRALDE UC, COSSON JP, DEBRE B, BOCCON-GIBOD L, STEG A.: Carcinome epidermoide de la vessie. A propos de quarante-six observations. Ann Urol 1987; 21: 179-182.

4. BADAWI AF, MOSTAFA MH, PROBERT A, O־CONNOR PJ.: Role of schistosomiasis in human bladder cancer: evidence of association, aetiological factors, and basic mechanisms of carcinogenesis. Eur $J$ Cancer Prevention 1995; 4: 45-49.

5. FORTUNY J, KOGEVINAS M, CHANG-CLAUDE $\mathrm{J}$ y cols.: Tobacco, occupation and non-transitional-cell carcinoma of the bladder: an international case-control study. Int J Cancer 1999; 80: 44-46.
6. ZAIDI SZ, THEAKER JM, SMART CJ.: Squamous cell carcinoma in a patient on clean intermitent self-catheterization (case report). BrJUrol 1997; 80: 352-353.

7. BICKELA, CULKIN DJ, WHEEJER JS.: Bladder cancer in spinal cord injury patients. J Urol 1991; 146: 1.240-1.242.

8. SAKAMOTO N, TSUNEYOSHI M, ENJOJI M.: Urinary bladder carcinoma with a neoplastic squamous component. A mapping study of 31 cases. Histopatology 1992; 21: 135-141.

9. NAVON JD, SOLIMAN H, KHONSARI F, AHLERING T.: Screening cystoscopy and survival of spinal cord injured patients with squamous cell cancer of the bladder. $J$ Urol 1997; 157: 2.109-2.111.

10. DEBBAGH A, BENNANI S, HAFIANI M, EL-MRINI M, BENJELLOUN S.: Epidermoid carcinoma of the bladder: a propos of 14 cases. Ann Urol (Paris) 1997; 31: 199-203.

11. ASAMOTO M, FUKUSHIMA S, TATEMOTO Y, YAMADA K, FUKUI S, MORI M.: Immunohistochemical expresion of keratin proteins in urinary bladder carcinoma. Pathol Res Pract 1989; 184: 194-201.

12. ASAMOTO M, FUKUSHIMA S, TATEMOTO Y, YAMADA K, YUBA R, MORI M.: Immunohistochemical evaluation of nonspecific cross reactive antigen and carcinoembryonic antigen (CEA) in urinary bladder carcinoma. Anticancer Res 1989; 9: 319-326.

13. BUDIA ALBA A, QUEIPO ZARAGOZA JA, PEREZ EBRÍ ML y cols.: Estudio comparativo del carcinoma epidermoide de vejiga puro y el carcinoma transicional con focos de diferenciación escamosa o mixto. Actas Urol Esp 1999; 23: 111-118.

14. GHONEIM MA, EL-MEKRESH MM, EL-BAZ MA, ELATTAR IA, ASHAMALLAH A.: Radical cystectomy for carcinoma of the bladder: critical evaluation of the results in 1,026 cases. J Urol 1997; 158: 393-399.

15. SWANSON DA, LILES A, ZAGARS GK.: Preoperative irradiation and radical cystectomy for stages $\mathrm{T} 2$ and T3 squamous cell carcinoma of the bladder. $J$ Urol 1990; 143: 37-40.

16. PATTERSON JM, RAY EH, MENDIONDO OA, MEDINA WD, GEE WF.: A new treatment for invasive squamous cell bladder. The Nigro regimen: preoperative chemotherapy and radiation therapy. $J$ Urol 1988; 140: 379-380.

17. GHONEIM MA, ASHAMALLAH AK, AWAAD HK, WHITMORE WF.: Randomized trial of cystectomy with or without preoperative radiotherapy for carcinoma of the bilharzial bladder. J Urol 1985; 134: 266-268.

18. UEKI O, HISAZUMI H, UCHIBAYASHI T y cols.: Metotrexate, vinblastine doxorubicin, and cisplatin for advanced urothelial cancer. Cancer Chemother Pharmacol 1992; 30: 72-76.

19. DANDEKAR NP, DALAL AV, TONGAONKAR HB, KAMAT MR.: Adenocarcinoma of bladder. Eur J Surg Oncol 1997; 23: 157-160.

20. PEGORARO V, COSCIANI-CUNICO S, GRAZIOTTI P, DALLA PALMA P.: L'adenocarcinoma mesonephrique de la vessie. J d'Urol 1982; 88: 531-532.

21. SÁNCHEZ ZALABARDO D, RODRÍGUEZ GONZÁLEZ J, FERNÁNDEZ MONTERO JM y cols.: Adenocarcinoma vesical primario: nuestra experiencia en los últimos 10 años. Actas Urol Esp 2001; 25: 573577. 
22. SALINAS SÁNCHEZ A, ALCALÁ-SANTAELLA CASANOVA C, MARTÍNEZ MARTÍN M, PASTOR GUZMÁN JM, CAÑAMARES PABOLAZA L, VIRSEDA RODRÍGUEZ J.: Adenocarcinomas de Uraco. Arch Esp de Urol 1991; 44: 31-36.

23. BENNETT JK, WHEATLEY JK, WALTON KN.: 10year experience with adenocarcinoma of the bladder. J Urol 1984; 131: 262-263.

24. FITER L, GIMENO F, MARTÍN L, GÓMEZ-TEJADA L.: Signet-ring cell adenocarcinoma of the bladder. Urology 1993; 41: 30-33.

25. GRIGNON DJ, RO JY, AYALA AG, JOHNSON DE, ORDÓNEZZ NG.: Primary adenocarcinoma of the bladder. Cancer 1991; 67: 2.165-2.172.

26. FERNÁNDEZ A, MAGANTO E, GARCÍA R, VALLEJO I, ESCUDERO A.: Adenocarcinoma vesical primario tipo células en anillo de sello. Arch Esp Urol 1990; 43: 355-358.

27. VARO SOLÍS C, BÁEZ PEREA JM, GARRIDO INSÚA S, BELTRÁN AGUILAR V.: Neoplasia de células en anillo de sello. Una variedad histológica rara como tumor vesical primario. Actas Urol Esp 2002; 26: 297-301.

28. KAMAT MR, KULKARNI JN, TONGAONKAR B.: Adenocarcinoma of the bladder: study of 14 cases and review of the literature. Br J Urol 1991; 68: 254257.

29. FERNÁNDEZ A, MAGANTO E, VALLEJO J, ESCUDERO A.: Adenocarcinoma vesical primario como tumor único. Estudio de siete casos y revisión de la literatura. Actas Urol Esp 1990; 14: 18-22.

30. EBLE JN, YOUNG RH.: Carcinoma of the urinary bladder: a review of its diverse morphology. Semin Diagn Pathol 1997; 14: 98-108.
31. ANDERSTROM C, JOHANSSON SL, SCHULTZ L.: Primary adenocarcinoma of the urinary bladder. A clinicopathologic and prognostic study. Cancer 1983; 52: 1.273-1.280.

32. RICOS JV, ALMENAR S, IBORRA I y cols.: Carcinosarcoma vesical. A propósito de dos casos. Arch Esp Urol 1993; 46: 140-143.

33. OLIVER C, LAO AH, MONLLOR J, CARALLIDO J.: Carcinosarcoma vesical con diferenciación osteosarcomatosa. Arch Esp Urol 1991; 15: 375-380.

34. LAHOTI C, SCHINELLA R, RANGWALA AF, LEE M, MIZRACHI H.: Carcinosarcoma of urinary bladder: report of 5 cases with immunohistologic study. Urology 1994; 43: 389-393.

35. IKEGAMI H, IWASAKI H, OHJIMI Y, TAKEUCHI T, ARIYOSHI A, KIKUCHI M.: Sarcomatoid carcinoma of the urinary bladder: a clinicopathologic and immunohistochemical analysis of 14 patients. Hum Pathol 2000; 31: 332-340.

36. SEN SE, MALEK RS, FARROW GM, LEIBER MM.: Sarcoma and carcinosarcoma of the bladder. $J$ Urol 1985; 133: 29-30.

37. GIANNOPOULOS A, ALIVIZATOS G, KYRIAKOU V, MITROPOULOS D, DIMOPOULOS MA.: Carcinosarcoma of the bladder. Br J Urol 1991; 67: 106-107.

Dr. J.A. Queipo Zaragozaá

C/ Santa Teresa, 4-8

46530 Puzol (Valencia)

(Trabajo recibido el 27 junio de 2002) 